Article

\title{
Global Stability of Integral Manifolds for Reaction-Diffusion Delayed Neural Networks of Cohen-Grossberg-Type under Variable Impulsive Perturbations
}

\author{
Gani Stamov ${ }^{1, *}$, Ivanka Stamova ${ }^{2}$, George Venkov ${ }^{3}$, Trayan Stamov ${ }^{4}$ and Cvetelina Spirova ${ }^{1}$ \\ 1 Department of Mathematical Physics, Technical University of Sofia, 8800 Sliven, Bulgaria; \\ cvetelina2802@abv.bg \\ 2 Department of Mathematics, University of Texas at San Antonio, San Antonio, TX 78249, USA; \\ ivanka.stamova@utsa.edu \\ 3 Department of Mathematical Analysis and Differential Equations, Technical University of Sofia, \\ 1000 Sofia, Bulgaria; gvenkov@tu-sofia.bg \\ 4 Department of Machine Elements and Non-metallic Constructions, Technical University of Sofia, \\ 1000 Sofia, Bulgaria; tstamov@tu-sofia.bg \\ * Correspondence: stamov@tu-sofia.bg
}

Received: 11 May 2020; Accepted: 30 June 2020; Published: 3 July 2020

check for updates

\begin{abstract}
The present paper introduces the concept of integral manifolds for a class of delayed impulsive neural networks of Cohen-Grossberg-type with reaction-diffusion terms. We establish new existence and boundedness results for general types of integral manifolds with respect to the system under consideration. Based on the Lyapunov functions technique and Poincarè-type inequality some new global stability criteria are also proposed in our research. In addition, we consider the case when the impulsive jumps are not realized at fixed instants. Instead, we investigate a system under variable impulsive perturbations. Finally, examples are given to demonstrate the efficiency and applicability of the obtained results.
\end{abstract}

Keywords: integral manifolds; Cohen-Grossberg-type neural networks; delays; reaction-diffusion terms; variable impulsive perturbations; boundedness; stability

\section{Introduction}

The neural network models of Cohen-Grossberg type have been initially introduced in 1983 [1]. Since the above pioneered publication, the theory and applications of the Cohen-Grossberg neural networks (CGNNs) have been developed in numerous research papers [2-4]. In addition, delayed CGNNs have been massively investigated due to their enormous opportunities of applications in diverse areas of science and engineering [5-10].

Moreover, many researchers considered the effect of reaction-diffusion terms on the dynamic behavior of neural networks [11-15]. Indeed, diffusion effects are essential in modelling and scientific understanding of natural and artificial neural networks. The properties of the hybrid type of CGNNs with reaction-diffusion terms have been also widely investigated in the existing literature [16-19].

In addition, the effect of various types of impulsive perturbations such as those at fixed moments of time, at variable times or delayed impulsive perturbations has been found to be remarkably important in the behavior and control of numerous systems. That is why impulsive differential equations and impulsive control systems are intensively used as tools in modelling of processes studied in widespread areas of the mathematical, physical, chemical, engineering, and statistical sciences [20-29]. 
Because of the boundless opportunities for modelling applications, a growing activity in the study of impulsive CGNNs with and without reaction-diffusion term has been noted in recent years. Many important results focused on the qualitative properties of such models increasingly appear in the literature. We will direct the reader to [30-40], and the references therein, for example.

However, in most of the existing results for impulsive CGNNs, the authors investigated the behavior of single states of interest. For example, in [30] the authors studied some fundamental and stability properties of asymptotic almost automorphic solutions for impulsive delayed CGNNs. The behavior of almost periodic states for delayed CGNNs under variable impulsive perturbations has been investigated in [31]. Fundamental and stability properties of the equilibrium point of delayed CGNNs under impulsive control are the subject of interest in [32]. The global exponential stability behavior of periodic solutions for delayed impulsive CGNNs with two layers of neurons is studied in [33]. In [34,35,37,40] different sufficient conditions for the fundamental and global exponential stability properties of equilibrium points for delayed impulsive CGNNs with reaction-diffusion terms are established. In [36] the global exponential stability behavior of anti-periodic solutions to impulsive CGNNs on time scales is mainly investigated. In [38] the authors are interested in the study of the single periodic solution for higher-order delayed CGNNs with impulses. The global exponential stability of equilibrium points of delayed impulsive CGNNs with diffusion is the subject of investigation in [39].

In the proposed paper, we consider the behavior of integral manifolds instead of single solutions. This is the first important novelty in our research. For a class of reaction-diffusion impulsive CGNNs with time-varying delays we introduce the concept of integral manifolds. In fact, the integral manifolds methods are powerful tools in the qualitative theory of different types of systems and that is why, they are of great interest in connection with numerous problems in physics and engineering [41-48]. It is reasonable that the analysis of integral manifolds and their behavior is one of the most interesting problems, and it is not yet developed for neural network models. In the present paper, we study the fundamental and qualitative properties of integral manifolds related to reaction-diffusion impulsive CGNNs with time-varying delays, namely existence, boundedness, global asymptotic and exponential stability which is the second contribution of our research.

The third highlight of this paper includes the consideration of variable impulsive perturbations in our analysis which are not considered in the existing results on impulsive CGNNs [30-40]. Instantaneous changes at not fixed instants of time have a considerable impact on the dynamics of the systems and are more general, hence, more important for applications [49-52]. However, the study of systems with variable impulsive perturbations is more challenging because of the existing opportunities for loss of the property of autonomy, bifurcation, "merging" of solutions, "beating" phenomena, etc. $[27,49]$. The impact of variable impulsive perturbations on the almost periodicity of delayed impulsive CGNNs has been investigated in [31]. However, the class of impulsive CGNNs in [31] does not include reaction-diffusion terms. In addition, the integral manifolds methods are not applied in the above paper. In the present article, we will expand and complement the pioneered stability results in [31] to the integral manifolds case and considering reaction-diffusion terms.

The fourth contribution of our research is devoted to the application of a Poincarè-type integral inequality [53] which will allow us a more accurate estimation of the reaction diffusion terms, and leads to a better exploration of the diffusion effect on the qualitative behavior of the considered class of impulsive CGNNs. See [54], and the references therein.

The plan of the rest of the manuscript is as follows. In Section 2, for a class of delayed reaction-diffusion CGNN models under variable impulsive perturbations, we will introduce the concept of integral manifolds. Some basic notations, definitions and comparison results are also given. Section 3 is devoted to existence and boundedness results for integral manifolds associated with the introduced in Section 2 model. In Section 4, integral manifolds global stability analysis is conducted and new criteria are proved. In Section 5, we present two examples to demonstrate the applicability of the the proposed existence, boundedness and exponential stability criteria. Finally, conclusions are given in Section 6. 


\section{Preliminaries}

Let $\mathbb{R}_{+}=[0, \infty),\|x\|=\sum_{q=1}^{n}\left|x_{q}\right|$ be the norm of $x=\left(x_{1}, x_{2}, \ldots, x_{n}\right)^{T} \in \mathbb{R}^{n}$. In $\mathbb{R}^{n}$ consider an open and bounded set $\Theta$ that has smooth boundary $\partial \Theta$ and the measure is expressed by mes $\Theta>0$. Let $\mathbf{0}=(0,0, \ldots, 0)^{T} \in \Theta$.

In this paper we will introduce the integral manifolds approach to the following CGNN model with reaction-diffusion terms and time-varying delays under variable impulsive perturbations

$$
\left\{\begin{aligned}
\frac{\partial u_{i}(t, x)}{\partial t}= & \sum_{q=1}^{n} \frac{\partial}{\partial x_{q}}\left(D_{i q} \frac{\partial u_{i}(t, x)}{\partial x_{q}}\right)-a_{i}\left(u_{i}(t, x)\right)\left[b_{i}\left(u_{i}(t, x)\right)\right. \\
& -\sum_{j=1}^{m} c_{i j}(t) f_{j}\left(u_{j}(t, x)\right) \\
& \left.-\sum_{j=1}^{m} w_{i j}(t) g_{j}\left(u_{j}\left(t-s_{j}(t), x\right)\right)\right], t \neq \tau_{k}\left(u_{i}(t, x)\right), i=1,2, \ldots, m, k=1,2, \ldots, \\
\Delta u_{i}(t, x)= & J_{i k}\left(u_{i}(t, x)\right), t=\tau_{k}\left(u_{i}(t, x)\right), i=1,2, \ldots, m, k=1,2, \ldots,
\end{aligned}\right.
$$

where:

a $\quad m, m \geq 2$ is the number of neurons in the GCNN model, $t>0, x=\left(x_{1}, x_{2}, \ldots, x_{n}\right)^{T} \in$ $\Theta, u_{i}(t, x)$ denotes the state of the $i$-th neuron at time $t$ and in space $x, u(t, x)=$ $\left(u_{1}(t, x), u_{2}(t, x), \ldots, u_{m}(t, x)\right)^{T} \in \mathbb{R}^{m}$;

b $\quad s_{j}(t)$ denote the time-varying delays of the $i$-th neural unit, the functions $s_{j}$ are continuous and $t>s_{j}, j=1, \ldots, m, 0 \leq s_{j}(t) \leq v, \frac{d s_{j}(t)}{d t}<\delta_{j}\left(v>0, \delta_{j}<1\right) ;$

c $\quad a_{i}\left(u_{i}(t, x)\right) \geq 0, i=1,2, \ldots, m$, are the amplification functions and are continuous on their domains;

d $\quad b_{i}\left(u_{i}(t, x)\right)$ are appropriately behaved continuous functions with real values, $i=1,2, \ldots, m$;

e $\quad c_{i j}(t)$ and $w_{i j}(t)$ are the connection weight and time-varying delay connection weight of $j$-th neural unit on the $i$-th neural unit at time $t$, respectively, and are continuous real-valued functions;

f $f_{j}\left(u_{j}(t, x)\right)$ and $g_{j}\left(u_{j}\left(t-s_{j}(t), x\right)\right), j=1, \ldots, m$ are the activation function and time-varying delay activation function of the $j$-th neuron, respectively, and for any $j=1,2, \ldots, m$ the functions $f_{j}, g_{j}$ are continuous with real values;

g $\quad D_{i q}=D_{i q}(t, x) \geq 0, q=1,2, \ldots, n, i=1,2, \ldots, m$ are the diffusion coefficients along the $q$-th coordinate for $i$-th neural unit and are continuous functions for any $i=1,2, \ldots, m$ and $q=1,2, \ldots, n$.

$\mathrm{h} \quad J_{i k}\left(u_{i}(t, x)\right), i=1,2, \ldots, m, k=1,2, \ldots$, are the real-valued functions that characterize the weights of the impulsive perturbations on the $i$-th nodes at the at the variable times for $t=\tau_{k}(u(t, x))$, where $\tau_{k}(u)$ are continuous functions, $k=1,2, \ldots ; \Delta u_{i}(t, x)=u_{i}\left(t^{+}, x\right)-u_{i}\left(t^{-}, x\right)$ for any $i=1,2, \ldots, m, t=\tau_{k}\left(u_{i}(t, x)\right), k=1,2, \ldots, x \in \Theta$, where $u_{i}\left(t^{-}, x\right)=u_{i}(t, x)$ is the state of the $i$-th neuron before the jump perturbation at $t=\tau_{k}\left(u_{i}(t, x)\right)$ and $u_{i}\left(t^{+}, x\right)$ is the impulsively controlled output of the $i$-th unit.

In order to formulate the initial condition for the delayed model (1), we consider an initial function $\varphi_{0}=\left(\varphi_{01}, \varphi_{02}, \ldots, \varphi_{0 m}\right)^{T}$ with real-valued components $\varphi_{0 i}(s, x)$ defined on $[-v, 0] \times \Theta$, that are bounded and piecewise continuous functions with respect to their first variable $s$ with eventually finite number of points of discontinuity of the first kind $s \in[-v, 0]$ at which $\varphi_{0 i}\left(s^{+}, x\right)$ and $\varphi_{0 i}\left(s^{-}, x\right)$ exist and $\varphi_{0 i}\left(s^{-}, x\right)=\varphi_{0 i}(s, x), i=1,2, \ldots, m, x \in \Theta$. 
Denote by $u(t, x)=u\left(t, x ; \varphi_{0}\right)$ the solution of the delayed impulsive reaction-diffusion CGNN model (1) under the following initial and boundary conditions:

$$
\begin{gathered}
u_{i}(s, x)=\varphi_{0 i}(s, x), s \in[-v, 0], x \in \Theta, i=1,2, \ldots, m, \\
u_{i}(t, x)=0, t \in[-v, \infty), x \in \partial \Theta, i=1,2, \ldots, m .
\end{gathered}
$$

According to the theory $[27,31,49]$, the solution

$$
u(t, x)=\left(u_{1}(t, x), u_{2}(t, x), \ldots, u_{m}(t, x)\right)^{T}
$$

of the model (1) with variable impulsive perturbations is such that at the moments $t_{l_{k}}$ when the integral surface of $u(t, x)$ meets the hypersurfaces

$$
\sigma_{k}=\left\{(t, u) \in[0, \infty) \times \mathbb{R}^{m}: t=\tau_{k}(u(t, x))\right\},
$$

the following is true

$$
u_{i}\left(t_{l_{k}}^{-}, x\right)=u_{i}\left(t_{l_{k}}, x\right), u_{i}\left(t_{l_{k}}^{+}, x\right)=u_{i}\left(t_{l_{k}}, x\right)+J_{i l_{k}}\left(u_{i}\left(t_{l_{k}}, x\right)\right), x \in \Theta, i=1,2, \ldots, m, k=1,2, \ldots
$$

The above points $t_{l_{1}}, t_{l_{2}}, \ldots$ are the impulsive moments. It is also well known [27,31,49] that, in general, $k \neq l_{k}$. In addition, due to the nature of the variable impulsive perturbations, different nodes $u_{i}(t, x), i=1,2, \ldots, m$ may have different impulsive moments. In this paper, we will investigate such nodes the motion along with which is established by a suitable choice of the impulsive forces. That is why we will assume that, $\tau_{0}\left(u_{i}\right) \equiv t_{0}=0$ for $u_{i} \in \mathbb{R}, i=1,2, \ldots, m$, all functions $\tau_{k}\left(u_{i}\right), i=1,2, \ldots, m$, $k=1,2, \ldots$ are continuous and

$$
t_{0}<\tau_{1}\left(u_{i}\right)<\tau_{2}\left(u_{i}\right)<\ldots, \tau_{k}\left(u_{i}\right) \rightarrow \infty \text { as } k \rightarrow \infty
$$

uniformly on $u_{i} \in \mathbb{R}$.

Further, we will use the following classes of functions:

(i) The class of all nonnegative continuous functions defined on $\mathbb{R}_{+}$that are strictly increasing and are zeros at zero will be denoted by $\mathcal{K}$;

(ii) The class of all functions $\bar{\sigma}: \mathbb{R} \times \Theta \rightarrow \mathbb{R}^{m}$ that are continuous everywhere on their domains except at points of the type $\left(t_{l_{k}}, x\right) \in \mathbb{R} \times \Theta$ where $\bar{\sigma}\left(t_{l_{k}}^{-}, x\right)$ and $\bar{\sigma}\left(t_{l_{k}}^{+}, x\right)$ exist and $\bar{\sigma}\left(t_{l_{k}}^{-}, x\right)=$ $\bar{\sigma}\left(t_{l_{k}}, x\right)$ will be denoted by $P C\left[\mathbb{R} \times \Theta, \mathbb{R}^{m}\right]$;

(iii) $\quad \mathcal{P C}$ will denote the class of all piecewise continuous functions $\varphi=\left(\varphi_{1}, \varphi_{2}, \ldots, \varphi_{m}\right)^{T} \in \mathbb{R}^{m}$ defined on $[-v, 0] \times \Theta$ for which $\varphi_{i}\left(s^{+}, x\right), \varphi_{i}\left(s^{-}, x\right)$ exist, $\varphi_{i}\left(s^{-}, x\right)=\varphi_{i}(s, x), i=1,2 \ldots, m$, for all points $(s, x) \in[-v, 0] \times \Theta$ which must be finite number;

(iv) $\quad P C B$ will denote the class of all functions $\varphi \in \mathcal{P C}$ that are bounded on $[-v, 0] \times \Theta$.

For $t \in \mathbb{R}_{+}$, we also consider the following norm

$$
\|u(t, .)\|_{2}=\left[\int_{\Omega} \sum_{i=1}^{m} u_{i}^{2}(t, x) d x\right]^{1 / 2}
$$

for $u(t, x)=\left(u_{1}(t, x), u_{2}(t, x), \ldots, u_{m}(t, x)\right)^{T} \in \mathbb{R}^{m}$, and the norm $\|.\|_{v}$ defined as

$$
\|\varphi\|_{v}=\sup _{-v \leq s \leq 0}\|\varphi(s, .)\|_{2}
$$

for a function $\varphi \in \mathcal{P C}$. 
In this paper we will apply the method of integral manifolds for the delayed reaction-diffusion impulsive CGNN model (1). To this end, we will adopt the following definition for an integral manifold related to (1) [41-48].

Definition 1. We will say that a manifold $M$ in the extended phase space $[-v, \infty) \times \Theta \times \mathbb{R}^{m}$ of (1) is an integral manifold, if $\left(s, x, \varphi_{0}(s, x)\right) \in M,(s, x) \in[-v, 0] \times \Theta$ implies $(t, x, u(t, x)) \in M,(t, x) \in \mathbb{R}_{+} \times \Theta$ for any solution $u(t, x)=u\left(t ; x ; \varphi_{0}\right)$.

For a manifold $M \subset[-v, \infty) \times \Theta \times \mathbb{R}^{m}$ we introduce the following sets and distances:

The set of all $u \in \mathbb{R}^{m}$ such that $(t, x, u) \in M$ for $(t, x) \in \mathbb{R}_{+} \times \Theta$ is denoted by $M(t, x)$;

The set of all $z \in \mathbb{R}^{m}$ such that $(s, x, z) \in M$ for $(s, x) \in[-v, 0] \times \Theta$ is denoted by $M_{0}(s, x)$;

The distance between $u \in \mathbb{R}^{m}$ and $M(t, x)$ is defined as $d(u, M(t, x))=\inf _{v \in M(t, x)}\|u-v\|_{2}$;

An $\varepsilon$ - neighborhood of $M(t, x)$ is denoted by $M(t, x, \varepsilon)$ and is defined by

$$
M(t, x, \varepsilon)=\left\{u \in \mathbb{R}^{m}: d(u, M(t, x))<\varepsilon\right\}(\varepsilon>0) ;
$$

The distance between a function $\varphi \in \mathcal{P C}$ and $M_{0}(s, x)$ is defined as

$$
d_{0}\left(\varphi, M_{0}(s, x)\right)=\sup _{s \in[-v, 0]} d\left(\varphi(s, x), M_{0}(s, x)\right) ;
$$

An $\varepsilon$ - neighborhood of $M_{0}(s, x)$ is denoted by $M_{0}(s, x, \varepsilon)$ and is defined by

$$
M_{0}(s, x, \varepsilon)=\left\{\varphi \in \mathcal{P C}: d_{0}\left(\varphi, M_{0}(s, x)\right)<\varepsilon\right\} .
$$

We will also use the closures

$\bar{M}(t, x, \varepsilon)=\left\{u \in \mathbb{R}^{m}: d(u, M(t, x)) \leq \varepsilon\right\}$

$\overline{M_{0}}(s, x, \varepsilon)=\left\{\varphi \in \mathcal{P C}: d_{0}\left(\varphi, M_{0}(s, x)\right) \leq \varepsilon\right\} ;$

$\overline{S_{\alpha}}=\left\{u \in R^{m}:\|u\|_{2} \leq \alpha\right\} ; \overline{S_{\alpha}}(\mathcal{P C})=\left\{\varphi \in \mathcal{P C}:\|\varphi\|_{v} \leq \alpha\right\}$.

Throughout this paper, we will assume that:

A1. For the nonnegative continuous functions $a_{i}$ there exist constants $\underline{a}_{i}$ and $\bar{a}_{i}$ such that

$$
\underline{a}_{i} \leq a_{i}(\chi) \leq \bar{a}_{i}, \chi \in \mathbb{R},
$$

$i=1,2, \ldots, m$.

A2. For the continuous functions $b_{i}$ there exist positive constants $B_{i}$ with such that

$$
\frac{b_{i}\left(\chi_{1}\right)-b_{i}\left(\chi_{2}\right)}{\chi_{1}-\chi_{2}} \geq B_{i}>0
$$

for $\chi_{1}, \chi_{2} \in \mathbb{R}, \chi_{1} \neq \chi_{2}, i=1,2, \ldots, m$.

A3. The continuous functions $f_{j}$ and $g_{j}$ are bounded, there exist positive constants $L_{j}, M_{j}, j=$ $1,2, \ldots, m$, such that

$$
\begin{aligned}
& \left|f_{j}\left(\chi_{1}\right)-f_{j}\left(\chi_{2}\right)\right| \leq L_{j}\left|\chi_{1}-\chi_{2}\right|, \\
& \left|g_{j}\left(\chi_{1}\right)-g_{j}\left(\chi_{2}\right)\right| \leq M_{j}\left|\chi_{1}-\chi_{2}\right|
\end{aligned}
$$

for all $\chi_{1}, \chi_{2} \in \mathbb{R}, \chi_{1} \neq \chi_{2}$, and $f_{j}(0)=g_{j}(0)=0$ for any $j=1,2, \ldots, m$.

A4. For the continuous functions $D_{i q}$ there exist constants $d_{i q} \geq 0$ such that

$$
D_{i q}(t, x) \geq d_{i q}, t>0, x \in \Theta,
$$

for any $i=1,2, \ldots, m$ and $q=1,2, \ldots, n$. 
A5. The impulsive functions $J_{i k}$ are continuous and $J_{i k}(0)=0$ for all $i=1,2, \ldots, m$ and $k=1,2, \ldots$ A6. The sets $M(t, x)$ and $M_{0}(s, x)$ are nonempty for $(t, x) \in \mathbb{R}_{+} \times \Theta$ and $(s, x) \in$ $[-v, 0) \times \Theta$, respectively.

Next, the following boundedness and stability definitions for an integral manifold $M \subset[-v, \infty) \times$ $\Theta \times \mathbb{R}^{m}$ are introduced. They generalize and extend the known boundedness and stability definitions for impulsive delayed CGNNs with reaction-diffusion terms used in $[34,35,37,39,40]$ to the integral manifolds case.

Definition 2. We will say that an integral manifold $M$ of system (1) is:

(a) equi-bounded, if

$$
\begin{gathered}
(\forall \eta>0)(\forall \alpha>0)(\exists b=b(\eta, \alpha)>0) \\
\left(\forall \varphi_{0} \in \overline{S_{\alpha}}(\mathcal{P C}) \cap \overline{M_{0}}(s, x, \eta)\right)(\forall t \geq 0, \forall x \in \Theta): u\left(t, x ; \varphi_{0}\right) \in M(t, x, b) ;
\end{gathered}
$$

(b) uniformly bounded, if the number $b$ from (a) is independent of $\alpha>0$.

Definition 3. We will say that an integral manifold $M$ of system (1) is said to be:

(a) stable, if

$$
\begin{gathered}
(\forall \varepsilon>0)(\forall \alpha>0)(\exists \delta=\delta(\alpha, \varepsilon)>0) \\
\left(\forall \varphi_{0} \in \overline{S_{\alpha}}(\mathcal{P C}) \cap \overline{M_{0}}(s, x, \delta)\right)(\forall t \geq 0, \forall x \in \Theta): u\left(t, x ; \varphi_{0}\right) \in M(t, x, \varepsilon) ;
\end{gathered}
$$

(b) uniformly stable, if the number $\delta$ from (a) is independent of $\alpha>0$;

(c) uniformly globally asymptotically stable, if it is a uniformly stable, uniformly bounded, and

$$
\begin{gathered}
(\forall \varepsilon>0)(\forall \eta>0)(\exists T=T(\eta, \varepsilon)>0) \\
\left(\forall \varphi_{0} \in P C B\right)(\forall t \geq T, \forall x \in \Theta): u\left(t, x ; \varphi_{0}\right) \in M(t, x, \varepsilon) ;
\end{gathered}
$$

(d) uniformly globally exponentially stable, if

$$
\begin{gathered}
(\exists k>0)(\exists \gamma \geq 0)\left(\forall \varphi_{0} \in P C B\right)(\forall t \geq 0, \forall x \in \Theta): \\
u\left(t, x ; \varphi_{0}\right) \in M\left(t, x, k d_{0}\left(\varphi_{0}, M_{0}(s, x)\right) e^{-\gamma t}\right) .
\end{gathered}
$$

It is clear that, in particular cases, Definitions 2 and 3 can be used to investigate the behavior of single solutions, such as, zero states, equilibria, periodic solutions, etc. In such cases, the integral manifolds contain only the corresponding solutions. Note that such a generalization of the concepts for systems with variable impulsive perturbations is not trivial, since for such systems [31,49-52], different states may have different impulsive moments.

In addition, the introduced definitions extend the opportunities for applications of the integral manifolds methods [41-48] to specific systems studied in numerous areas of science and technologies, such as impulsive CGNNs with reaction diffusion terms and time-varying delays.

Next Poincarè-type integral inequality [54] for the set $\Theta=\prod_{q=1}^{n}\left[a_{q}, b_{q}\right], a_{q}=$ const $\in \mathbb{R}, b_{q}=$ const $\in \mathbb{R}, q=1,2, \ldots, n$ will be applied in the proofs of our main results.

Lemma 1. [53,54] For any real-valued function $v(x)$ that belongs to $C^{1}(\Theta)$ the following relation is valid

$$
\int_{\Theta} v^{2}(x) d x \leq \frac{B^{2}}{4 n} \int_{\Theta}|\nabla v(x)|^{2} d x
$$

whenever $\Theta=\prod_{q=1}^{n}\left[a_{q}, b_{q}\right], a_{q}, b_{q} \in \mathbb{R}, \mathbf{0}=(0,0, \ldots, 0)^{T} \in \Theta$ and $B=\max \left\{b_{q}-a_{q}, q=1,2, \ldots, n\right\}$. 
Some generalizations of Lemma 1 also exist in the literature. See, for example, [16].

Finally, some basic notations and results from the method of piecewise continuous Lyapunov-type functions $[6,11,13,15,27,28,31,35]$ are in order.

Define the sets

$$
\mathcal{G}_{k}=\left\{(t, u): \tau_{k-1}(u)<t<\tau_{k}(u), u \in \mathbb{R}^{m}\right\}, k=1,2, \ldots, \mathcal{G}=\bigcup_{k=1}^{\infty} \mathcal{G}_{k} .
$$

We will use Lyapunov-type functions from the class $V_{M}=\left\{V: \mathbb{R}_{+} \times \mathbb{R}^{m} \rightarrow \mathbb{R}_{+}: \quad V \in\right.$ $C(\mathcal{G}), V$ is locally Lipschitz with respect to its second argument on each of the sets $\mathcal{G}_{k}, V(t, u(t,))=$. 0 for $(t, x, u) \in M, V(t, u(t,))>$.0 for $(t, x, u) \in\left\{\mathbb{R}_{+} \times \Theta \times \mathbb{R}^{m}\right\} \backslash M$, for each $k=$ $1,2, \ldots$ and $\left(t_{0}^{*}, u_{0}^{*}\right) \in \sigma_{k}, V\left(t_{0}^{*-}, u_{0}^{*}\right)=V\left(t_{0}^{*}, u_{0}^{*}\right)$ and $V\left(t_{0}^{*+}, u_{0}^{*}\right)$ exist $\}$.

Denote by $t_{1}, t_{2}, \ldots\left(0=t_{0}<t_{1}<t_{2}<\ldots\right)$ the impulsive moments of the solution $u\left(t, x ; \varphi_{0}\right)$ of the problem (1), (2), (3). In fact, each of the points $t_{k}$ is a solution of some of the equations $t=\tau_{k}(u(t, x))$, $t \geq 0, x \in \Theta, k=1,2, \ldots$, i.e., $t_{k}$ are the impulsive points at which the integral curve $\left(t, u\left(t, x ; \varphi_{0}\right)\right)$ of the initial value boundary problem (1), (2) (3) meets each of the hypersurfaces $\sigma_{k}, k=1,2, \ldots$

Now, for a given function $V \in V_{M}, t \in \mathbb{R}_{+}, t \neq t_{k}, k=1,2, \ldots$ and $\bar{\varphi} \in \mathcal{P C}$ define the following derivative with respect to system (1)

$$
D^{+} V(t, \bar{\varphi}(0, .))=\lim _{\chi \rightarrow 0^{+}} \sup \frac{1}{\chi}[V(t+\chi, u(t+\chi, . ; \bar{\varphi}(0, .))-V(t, \bar{\varphi}(0, .))] .
$$

We will use the following key lemma.

Lemma 2. [31] Let assumptions $A 1-A 6$ hold, and a function $V \in V_{M}$ exists such that for $t \in \mathbb{R}_{+}$and $\varphi \in \mathcal{P C}$

$$
D^{+} V(t, \varphi(0, .)) \leq \lambda_{1} V(t, \varphi(0, .)), t \neq t_{k}, \lambda_{1} \in \mathbb{R}
$$

for

$$
V(t+s, \varphi(s, .)) \leq V(t, \varphi(0, .)),-v \leq s \leq 0
$$

and

$$
V\left(t^{+}, \varphi(0, .)+\Delta(\varphi, .)\right) \leq V(t, \varphi(0, .)), t=t_{k}, k=1,2, \ldots
$$

Then

$$
V(t, u(t, .)) \leq \sup _{-v \leq s \leq 0} V\left(0, \varphi_{0}(s, .)\right) e^{\lambda_{1} t}, t \geq 0 .
$$

\section{Existence and Boundedness Results}

In this section, existence and boundedness of an integral manifold with respect to system (1) will be investigated.

Let $a_{q}, b_{q} \in \mathbb{R}, q=1,2, \ldots, n$, and consider the set $\Theta$ of points $x, x=\left(x_{1}, x_{2}, \ldots, x_{n}\right)^{T}$ defined as $a_{q} \leq x_{q} \leq b_{q}$, i.e., $\Theta=\prod_{q=1}^{n}\left[a_{q}, b_{q}\right]$. Let $\mathbf{0}=(0,0, \ldots, 0)^{T} \in \Theta$.

Denote by $B=\max \left\{b_{q}-a_{q}\right\}, \underline{D}=\min \left\{d_{i q}\right\}$, and $\bar{c}_{i j}=\sup _{t \in \mathbb{R}_{+}}\left|c_{i j}(t)\right|, \bar{w}_{i j}=\sup _{t \in \mathbb{R}_{+}}\left|w_{i j}(t)\right|, i, j=$ $1,2, \ldots, m, q=1,2, \ldots, n$,

Introduce the following assumption:

A7. The impulsive controllers $J_{i k}$ of system (1) are designed such that

$$
J_{i k}\left(u_{i}\left(t_{k}, x\right)\right)=-\gamma_{i k} u_{i}\left(t_{k}, x\right), 0<\gamma_{i k}<2, i=1,2, \ldots, m, k=1,2, \ldots
$$


Theorem 1. A manifold $M$ in the extended phase space of the system (1) is an integral manifold of (1), if assumptions $A 1-A 7$ hold and the system's parameters are such that

$$
\min _{1 \leq i \leq m}\left[2\left(\frac{4 n \underline{D}}{B^{2}}+\underline{a}_{i} B_{i}\right)-\bar{a}_{i} \sum_{j=1}^{m}\left(L_{j} \bar{c}_{i j}+M_{j} \bar{w}_{i j}+L_{i} \bar{c}_{j i}\right)\right]>\max _{1 \leq i \leq m}\left(M_{i} \sum_{j=1}^{m} \bar{a}_{j} \bar{w}_{j i}\right)>0 .
$$

Proof. Let $x \in \Theta, \varphi_{0} \in P C B$ and $u(t, x)=u\left(t, x ; \varphi_{0}\right)=\left(u_{1}(t, x), u_{2}(t, x), \ldots, u_{m}(t, x)\right)^{T}$ be the solution of the initial value boundary problem (1), (2), (3) defined for $t \geq 0[34,35,37,39,40]$.

Assumption A6 guarantees the existence of at least one

$$
v=v(t, x)=\left(v_{1}(t, x), v_{2}(t, x), \ldots, v_{m}(t, x)\right)^{T} \in M(t, x),
$$

$(t, x) \in \mathbb{R}_{+} \times \Theta$.

We construct a Lyapunov-type function $V \in V_{M}$ as follows

$$
V(t, u)=\frac{1}{2} d^{2}(u, M(t, x))=\frac{1}{2} \inf _{v \in M(t, x)}\|u(t, .)-v(t, .)\|_{2}^{2},(t, u) \in \mathbb{R}_{+} \times \mathbb{R}^{m}
$$

or

$$
V(t, u)=\frac{1}{2} \inf _{v \in M(t, x)} \int_{\Theta} \sum_{i=1}^{m}\left(u_{i}(t, x)-v_{i}(t, x)\right)^{2} d x,(t, u) \in \mathbb{R}_{+} \times \mathbb{R}^{m} .
$$

Since for any $t \in \sigma_{k}, k=1,2, \ldots, \mathrm{A} 7$ implies

$$
\int_{\Theta} \sum_{i=1}^{m}\left(1-\gamma_{i k}\right)^{2}\left(u_{i}(t, x)-v_{i}(t, x)\right)^{2} d x<\int_{\Theta} \sum_{i=1}^{m}\left(u_{i}(t, x)-v_{i}(t, x)\right)^{2} d x
$$

then, for any $\varphi \in \mathcal{P C}$ we have

$$
V\left(t^{+}, \varphi(0, .)+J_{k}(\varphi, .)\right)<V(t, \varphi(0, .)), t=t_{k}, k=1,2, \ldots
$$

Now, for any $t \notin \sigma_{k}, k=1,2, \ldots$, for the derivative of the function $V(t, u(t,)$.$) , we have$

$$
\frac{d V(t, u(t, .))}{d t} \leq \sum_{i=1}^{m} \int_{\Theta}\left(u_{i}(t, x)-v_{i}(t, x)\right) \frac{\partial\left(u_{i}(t, x)-v_{i}(t, x)\right)}{\partial t} d x
$$

Since $v \in M(t, x)$, then by A1, we obtain

$$
\begin{aligned}
\frac{\partial\left(u_{i}(t, x)-v_{i}(t, x)\right)}{\partial t} & \leq \sum_{q=1}^{n} \frac{\partial}{\partial x_{q}}\left(D_{i q} \frac{\partial\left(u_{i}(t, x)-v_{i}(t, x)\right)}{\partial x_{q}}\right) \\
& -\underline{a}_{i}\left[b_{i}\left(u_{i}(t, x)\right)-b_{i}\left(v_{i}(t, x)\right)\right]+\bar{a}_{i} \sum_{j=1}^{m} \bar{c}_{i j}\left|f_{j}\left(u_{j}(t, x)\right)-f_{j}\left(v_{j}(t, x)\right)\right| \\
& +\bar{a}_{i} \sum_{j=1}^{m} \bar{w}_{i j}\left|g_{j}\left(u_{j}\left(t-s_{j}(t), x\right)\right)-g_{j}\left(v_{j}\left(t-s_{j}(t), x\right)\right)\right| .
\end{aligned}
$$


From (8), we get

$$
\begin{aligned}
& \int_{\Theta}\left(u_{i}(t, x)-v_{i}(t, x)\right) \frac{\partial\left(u_{i}(t, x)-v_{i}(t, x)\right)}{\partial t} \\
& \leq \int_{\Theta} \sum_{q=1}^{n} \frac{\partial}{\partial x_{q}}\left(D_{i q} \frac{\partial\left(u_{i}(t, x)-v_{i}(t, x)\right)}{\partial x_{q}}\right)\left(u_{i}(t, x)-v_{i}(t, x)\right) d x \\
& -\int_{\Theta} a_{i}\left(u_{i}(t, x)-v_{i}(t, x)\right)\left[b_{i}\left(u_{i}(t, x)\right)-b_{i}\left(v_{i}(t, x)\right)\right] d x \\
& +\bar{a}_{i} \int_{\Theta}\left(u_{i}(t, x)-v_{i}(t, x)\right) \sum_{j=1}^{m} \bar{c}_{i j}\left|f_{j}\left(u_{j}(t, x)\right)-f_{j}\left(v_{j}(t, x)\right)\right| d x \\
& +\bar{a}_{i} \int_{\Theta}\left(u_{i}(t, x)-v_{i}(t, x)\right) \sum_{j=1}^{m} \bar{w}_{i j}\left|g_{j}\left(u_{j}\left(t-s_{j}(t), x\right)\right)-g_{j}\left(v_{j}\left(t-s_{j}(t), x\right)\right)\right| d x .
\end{aligned}
$$

Now, we will estimate each term of the right-hand side of (9).

We apply the Green's theorem, the boundary conditions, A4 and Lemma 1 to obtain

$$
\begin{aligned}
& \int_{\Theta} \sum_{q=1}^{n} \frac{\partial}{\partial x_{q}}\left(D_{i q} \frac{\partial\left(u_{i}(t, x)-v_{i}(t, x)\right)}{\partial x_{q}}\right)\left(u_{i}(t, x)-v_{i}(t, x)\right) d x \\
& =-\sum_{q=1}^{n} \int_{\Theta} D_{i q}\left(\frac{\partial\left(u_{i}(t, x)-v_{i}(t, x)\right)}{\partial x_{q}}\right)^{2} d x \\
& \leq-\sum_{q=1}^{n} \int_{\Theta} d_{i q}\left(\frac{\partial\left(u_{i}(t, x)-v_{i}(t, x)\right)}{\partial x_{q}}\right)^{2} d x \leq-\frac{4 n \underline{D}}{B^{2}} \int_{\Theta}\left(u_{i}(t, x)-v_{i}(t, x)\right)^{2} d x .
\end{aligned}
$$

From A2 and A3, we obtain

$$
\begin{aligned}
\int_{\Theta} \underline{a}_{i}\left(u_{i}(t, x)-v_{i}(t, x)\right)\left[b_{i}\left(u_{i}(t, x)\right)-b_{i}\left(v_{i}(t, x)\right)\right] d x \geq \underline{a}_{i} B_{i} \int_{\Theta}\left(u_{i}(t, x)-v_{i}(t, x)\right)^{2} d x \\
\quad \bar{a}_{i} \int_{\Theta}\left(u_{i}(t, x)-v_{i}(t, x)\right) \sum_{j=1}^{m} \bar{c}_{i j}\left|f_{j}\left(u_{j}(t, x)\right)-f_{j}\left(v_{j}(t, x)\right)\right| d x \\
\leq \bar{a}_{i} \int_{\Theta} \sum_{j=1}^{m} \bar{c}_{i j} L_{j}\left|u_{i}(t, x)-v_{i}(t, x)\right|\left|u_{j}(t, x)-v_{j}(t, x)\right| d x \\
\leq \frac{1}{2} \bar{a}_{i} \sum_{j=1}^{m} \int_{\Theta} \bar{c}_{i j} L_{j}\left[\left(u_{i}(t, x)-v_{i}(t, x)\right)^{2}+\left(u_{j}(t, x)-v_{j}(t, x)\right)^{2}\right] d x
\end{aligned}
$$

and

$$
\begin{aligned}
& \bar{a}_{i} \int_{\Theta}\left(u_{i}(t, x)-v_{i}(t, x)\right) \sum_{j=1}^{m} \bar{w}_{i j}\left|g_{j}\left(u_{j}\left(t-s_{j}(t), x\right)\right)-g_{j}\left(v_{j}\left(t-s_{j}(t), x\right)\right)\right| d x \\
& \leq \bar{a}_{i} \sum_{j=1}^{m} \int_{\Theta} \bar{w}_{i j} M_{j}\left|u_{i}(t, x)-v_{i}(t, x)\right|\left|u_{j}\left(t-s_{j}(t), x\right)-v_{j}\left(t-s_{j}(t), x\right)\right| d x \\
& \leq \frac{1}{2} \bar{a}_{i} \sum_{j=1}^{m} \int_{\Theta} \bar{w}_{i j} M_{j}\left[\left(u_{i}(t, x)-v_{i}(t, x)\right)^{2}+\left(u_{j}\left(t-s_{j}(t), x\right)-v_{j}\left(t-s_{j}(t), x\right)\right)^{2}\right] d x .
\end{aligned}
$$


Now, from (7)-(13), we have

$$
\begin{aligned}
& \frac{d V(t, u(t, .))}{d t} \\
& \leq \sum_{i=1}^{m}\left[-\left(\frac{4 n \underline{D}}{B^{2}}+\underline{a}_{i} B_{i}\right) \int_{\Theta}\left(u_{i}(t, x)-v_{i}(t, x)\right)^{2} d x\right. \\
& +\frac{1}{2} \bar{a}_{i} \sum_{j=1}^{m} \int_{\Theta} \bar{c}_{i j} L_{j}\left[\left(u_{i}(t, x)-v_{i}(t, x)\right)^{2}+\left(u_{j}(t, x)-v_{j}(t, x)\right)^{2}\right] d x \\
& \left.+\frac{1}{2} \bar{a}_{i} \sum_{j=1}^{m} \int_{\Theta} \bar{w}_{i j} M_{j}\left[\left(u_{i}(t, x)-v_{i}(t, x)\right)^{2}+\left(u_{j}\left(t-s_{j}(t), x\right)-v_{j}\left(t-s_{j}(t), x\right)\right)^{2}\right] d x\right] \\
& \leq-\frac{1}{2} \sum_{i=1}^{m}\left[2\left(\frac{4 n \underline{D}}{B^{2}}+\underline{a}_{i} B_{i}\right)\right. \\
& \left.-\bar{a}_{i} \sum_{j=1}^{m}\left(L_{j} \bar{c}_{i j}+M_{j} \bar{w}_{i j}+L_{i} \bar{c}_{j i}\right)\right] \int_{\Theta}\left(u_{i}(t, x)-v_{i}(t, x)\right)^{2} d x \\
& +\frac{1}{2} \sum_{i=1}^{m} \sum_{j=1}^{m} \bar{a}_{j} \bar{w}_{j i} M_{i} \int_{\Theta} \sup _{-v \leq s \leq 0}\left(u_{j}(s, x)-v_{j}(s, x)\right)^{2} d x \\
& \leq-c_{1} \frac{1}{2} \sum_{j=1}^{m} \int_{\Theta}\left(u_{i}(t, x)-v_{i}(t, x)\right)^{2} d x+c_{2} \frac{1}{2} \sum_{j=1}^{m} \int_{\Theta} \underset{-v \leq s \leq 0}{\sup }\left(u_{j}(s, x)-v_{j}(s, x)\right)^{2} d x
\end{aligned}
$$

where

$$
\begin{gathered}
c_{1}=\min _{1 \leq i \leq m}\left[2\left(\frac{4 n \underline{D}}{B^{2}}+\underline{a}_{i} B_{i}\right)-\bar{a}_{i} \sum_{j=1}^{m}\left(L_{j} \bar{c}_{i j}+M_{j} \bar{w}_{i j}+L_{i} \bar{c}_{j i}\right)\right], \\
c_{2}=\max _{1 \leq i \leq m}\left(M_{i} \sum_{j=1}^{m} \bar{a}_{j} \bar{w}_{j i}\right) .
\end{gathered}
$$

It follows from (4) that

$$
D^{+} V(t, \varphi(0, .)) \leq 0, t \neq t_{k}
$$

for $\varphi \in \mathcal{P C}$ when $V(t+s, \varphi(s,).) \leq V(t, \varphi(0,)),.-v \leq s \leq 0$.

According to Lemma 2 for $\lambda_{1}=0$, by (6) and (15), we obtain

$$
V(t, u(t, .)) \leq \sup _{-v \leq s \leq 0} V\left(0, \varphi_{0}(s, .)\right), t \geq 0
$$

Now, for the solution $u\left(t, x ; \varphi_{0}\right)$ of the initial value boundary problem (1), (2), (3) we will prove that $\left(s, x, \varphi_{0}(s, x)\right) \in M$ for $(s, x) \in[-v, 0] \times \Theta$ implies $(t, x, u(t, x)) \in M,(t, x) \in \mathbb{R}_{+} \times \Theta$.

If the above assumption is not true, then for any $x \in \Theta$ there exists a $t^{\prime}, t^{\prime}>0$ such that $\left(t, x, u\left(t, x ; \varphi_{0}\right)\right) \in M$ for $0<t \leq t^{\prime}$ and $\left(t, x, u\left(t, x ; \varphi_{0}\right)\right) \notin M$ for $t>t^{\prime}$.

We consider the following cases:

Case 1. $t^{\prime} \notin \sigma_{k}$ for $k=1,2, \ldots$, or $t^{\prime} \neq \tau_{k}(u), u \in \mathbb{R}^{m}$, for $k=1,2, \ldots$ In this case, there exists $t^{\prime \prime}$, $\tau_{k}(u)<t^{\prime \prime}<\tau_{k+1}(u)$ such that $\left(t^{\prime \prime}, x, u\left(t^{\prime \prime}, x ; \varphi_{0}\right)\right) \notin M$ and

$$
V\left(t^{\prime \prime}, u\left(t^{\prime \prime}, x ; \varphi_{0}\right)\right)>0
$$

Then, for $t=t^{\prime \prime}$, from (16) since $\left(s, x, \varphi_{0}(s, x)\right) \in M$ for $(s, x) \in[-v, 0] \times \Theta$, we get $V\left(t^{\prime \prime}, u\left(t^{\prime \prime},.\right)\right) \leq \sup _{-v \leq s \leq 0} V\left(0, \varphi_{0}(s,).\right)=0$, which contradicts (17).

Case 2. $t^{\prime} \in \sigma_{k}$ for some $k=j, j+1, \ldots, j \geq 1$ or $t^{\prime}=\tau_{k}(u), u \in \mathbb{R}^{m}$, for some $k=j, j+1, \ldots, j \geq 1$. 
Then, from (6) and the fact that $\left(t^{\prime}, x, u\left(t^{\prime}, x ; \varphi_{0}\right)\right) \in M$, we have

$$
0 \leq V\left(t^{\prime+}, u\left(t^{\prime+}, .\right)\right)<V\left(t^{\prime}, u\left(t^{\prime}, .\right)\right)=0
$$

The contradictions obtained in both cases prove that $\left(s, x, \varphi_{0}(s, x)\right) \in M$ for $(s, x) \in[-v, 0] \times \Theta$ implies $(t, x, u(t, x)) \in M,(t, x) \in \mathbb{R}_{+} \times \Theta$, i.e., the set $M$ is an integral manifold for (1). Theorem 1 is proved.

Next is the boundedness result.

Theorem 2. Under the conditions of Theorem 1 the integral manifold M of model (1) is uniformly bounded.

Proof. For the integral manifold $M$, consider the Lyapunov-type function (5). We have that $V \in V_{M}$ and there exist $v_{1}, v_{2} \in \mathcal{K}$ such that

$$
v_{1}(d(u, M(t, x))) \leq V(t, u) \leq v_{2}(d(u, M(t, x))),(t, u) \in \mathbb{R}_{+} \times \mathbb{R}^{m}, x \in \Theta
$$

Let $\eta>0$ be chosen. It follows from $v_{1}, v_{2} \in \mathcal{K}$ that the number $b=b(\eta)>0$ can be chosen so that $v_{2}(\eta)<v_{1}(b)$.

Now, we suppose that $u(t, x)=u\left(t, x ; \varphi_{0}\right)$ is the solution of the problem (1), (2) and (3) with initial function $\varphi_{0} \in \overline{S_{\alpha}}\left(P C_{0}\right) \cap \overline{M_{0}}(s, x, \eta)$ for $\alpha>0$. From (18) and (16), we have

$$
\begin{gathered}
v_{1}(d(u(t, x), M(t, x))) \leq V(t, u(t, x)) \leq \sup _{-v \leq s \leq 0} V\left(0, \varphi_{0}(s, x)\right) \\
\leq v_{2}\left(d_{0}\left(\varphi_{0}, M_{0}(s, x)\right)\right) \leq v_{2}(\eta)<v_{1}(b), t \geq 0 .
\end{gathered}
$$

Therefore, $u\left(t, x ; \varphi_{0}\right) \in M(t, x, b)$ for $t \in \mathbb{R}_{+}$and the proof is completed.

\section{Integral Manifolds Stability Analysis}

In the next we will use measurable functions of the type $\lambda: \mathbb{R}_{+} \rightarrow \mathbb{R}_{+}$. Such functions are integrally positive if

$$
\int_{\hat{J}} \lambda(t) d t=\infty
$$

whenever $\hat{J}=\bigcup_{k=1}^{\infty}\left[\alpha_{k}, \beta_{k}\right], \alpha_{k}<\beta_{k}<\alpha_{k+1}$, and $\beta_{k}-\alpha_{k} \geq \mu>0, k=1,2, \ldots$

Theorem 3. If the conditions of Theorem 1 hold, and in addition, the integrally positive function $\lambda=\lambda(t)$ : $\mathbb{R}_{+} \rightarrow \mathbb{R}_{+}$is such that

$$
\min _{1 \leq i \leq m}\left[2\left(\frac{4 n \underline{D}}{B^{2}}+\underline{a}_{i} B_{i}\right)-\bar{a}_{i} \sum_{j=1}^{m}\left(L_{j} \bar{c}_{i j}+M_{j} \bar{w}_{i j}+L_{i} \bar{c}_{j i}\right)\right]-\max _{1 \leq i \leq m}\left(M_{i} \sum_{j=1}^{m} \bar{a}_{j} \bar{w}_{j i}\right)>\lambda(t) \geq 0
$$

for $t \neq \tau_{k}(u), k=1,2, \ldots$, then the integral manifold $M$ of the impulsive reaction-diffusion delayed CGNN (1) is uniformly globally asymptotically stable.

Proof. The uniform boundedness of the integral manifold $M$ follows from Theorem 2. Now, we will prove that $M$ is uniformly stable and globally attractive.

Consider the uniform stability. For the function $V \in V_{M}$ from Theorem 1, the inequalities (18) are true for functions $v_{1}, v_{2} \in \mathcal{K}$.

Then, for a given $\varepsilon>0$ there exists $\delta=\delta(\varepsilon)>0$ such that $v_{2}(\delta)<v_{1}(\varepsilon)$.

Consider a function $\varphi_{0} \in \overline{S_{\alpha}}(\mathcal{P C}) \cap M_{0}(t, x, \delta)$ for $\alpha>0$, and let $u\left(t, x ; \varphi_{0}\right)$ be the solution of the impulsive reaction-diffusion delayed CGNN (1) through $\left(0, \varphi_{0}\right)$. 
By condition (19) following the same steps as in the proof of Theorem 1 for $t \geq 0, t \neq \tau_{k}(u)$, $u \in \mathbb{R}^{m}$, for any function $\varphi \in \mathcal{P C}$ we get

$$
D^{+} V(t, \varphi(0, .)) \leq-\lambda(t) V(t, \varphi(0, .)), \quad t \neq \tau_{k}(u), t>0
$$

for $V(t+s, \varphi(s,).) \leq V(t, \varphi(0,)),.-v \leq s \leq 0$.

Using (6), (18)-(20), by Lemma 2, we get

$$
\begin{gathered}
v_{1}\left(d\left(u\left(t, x ; \varphi_{0}\right), M(t, x)\right)\right) \leq V(t, u(t, x)) \leq v_{2}\left(\sup _{-v \leq s \leq 0} V\left(0, \varphi_{0}(s, x)\right)\right) \\
\leq v_{2}\left(d\left(\varphi_{0}, M_{0}(s, x)\right)\right)<v_{2}(\delta)<v_{1}(\varepsilon), t \geq 0,
\end{gathered}
$$

hence $u\left(t, x ; \varphi_{0}\right) \in M(t, x, \varepsilon), t \geq 0$, which proves the uniform stability of the integral manifold $M$ of the model (1).

Finally, we will show that the integral manifold $M$ of model (1) is globally attractive. To this end, according to Definition 3(c), we will show that for the given $\eta>0$ and $\varepsilon>0$ we can take a constant $T=T(\eta, \varepsilon)>0$ so that for any $x \in \Theta$ the following inequality

$$
\sup _{t^{*}-v \leq t \leq t^{*}} d(u(t, x), M(t, x))<\delta(\varepsilon)
$$

is valid for at least one $t^{*} \in[0, T]$.

If (21) is not true for at least one $t^{*} \in[0, T]$, then

$$
d\left(u\left(t, x ; \varphi_{0}\right), M(t, x)\right) \geq \delta(\varepsilon)
$$

for any $(t, x) \in[0, T] \times \Theta$. In this case, by (6), (20) and (22), for any $t \in[0, T]$ it follows that

$$
\begin{aligned}
V(t, u(t, .))-V(0, u(0, .)) & \leq-\int_{0}^{t} \lambda(\vartheta) d(u(\vartheta, .), M(\vartheta, .)) d \vartheta \\
& \leq V(0, u(0, .))-\delta(\varepsilon) \int_{0}^{t} \lambda(\vartheta) d \vartheta
\end{aligned}
$$

Furthermore, from the integral positivity of the function $\lambda(t)$ we can choose the number $T$ so that

$$
\int_{0}^{T} \lambda(\vartheta) d \vartheta>\frac{v_{2}(\eta)}{\delta(\varepsilon)}
$$

Now, for $t=T$, from (23) and the uniform boundedness of the integral manifold $M$, we have

$$
V\left(T, u\left(T, x ; \varphi_{0}\right)\right) \leq v_{2}(\eta)-\delta(\varepsilon) \int_{0}^{T} \lambda(\vartheta) d \vartheta<0,
$$

which is a contradiction. Therefore, there exists a $t^{*} \in[0, T]$, such that the inequality (21) is satisfied.

Now, (6), (20) and the fact that $V$ is nonincreasing along the solution $u(t, x ; \varphi))$ of (1) imply that for $t \geq t^{*}$ we have

$$
\begin{gathered}
v_{1}\left(d\left(u\left(t, x ; \varphi_{0}\right), M(t, x)\right)\right) \leq V\left(t, u\left(t ; x, \varphi_{0}\right)\right) \leq v_{2}\left(\sup _{t^{*}-v \leq t \leq t^{*}} V\left(t^{*^{+}}, u(t, x)\right)\right) \\
=v_{2}\left(\sup _{t^{*}-v \leq t \leq t^{*}} d(u(t, x), M(t, x))\right)<v_{2}(\delta)<v_{1}(\varepsilon) .
\end{gathered}
$$

The above estimates are true for $t \geq T$ as well, and hence, $u(t, x) \in M(t, x, \epsilon), t \geq T$, which shows that the integral manifold $M$ of (1) is globally attractive. 
Theorem 4. If the conditions of Theorem 3 are met and there exists a constant $\lambda_{1}$ such that $\lambda(t) \geq \lambda_{1}>0$, $t \in \mathbb{R}_{+}$, then the integral manifold $M$ of (1) is globally exponentially stable.

Proof. Let $\varphi_{0} \in P C B$ and $u\left(t, x ; \varphi_{0}\right)$ be the solution of the initial value boundary problem (1), (2), (3). By Theorem 3, for the Lyapunov function $V \in V_{M}$, we get (6) and (20). Then, by Lemma 2, and $\lambda(t) \geq$ $\lambda_{1}>0, t \in \mathbb{R}_{+}$, we get

$$
V(t, u(t, .)) \leq \sup _{-v \leq s \leq 0} V\left(0, \varphi_{0}(s, .)\right) e^{-\lambda_{1} t}, t \geq 0 .
$$

Next, for the Lyapunov function $V \in V_{M}$ defined by (5), we have

$$
V(t, u)<k_{1} d^{2}(u, M(t, x)),(t, u) \in \mathbb{R}_{+} \times \mathbb{R}^{m}
$$

for a constant $k_{1}>\frac{1}{2}>0$.

Then from the choice of $V \in V_{M}$, conditions of Theorem 4, (24) and (25), we obtain

$$
\begin{aligned}
d\left(u\left(t, x ; \varphi_{0}\right), M(t, x)\right) & \leq(2 V(t, u(t, x)))^{1 / 2} \leq\left(2 \sup _{-v \leq s \leq 0} V\left(0, \varphi_{0}(s, .)\right) e^{-\lambda_{1} t}\right)^{1 / 2} \\
& <\sqrt{2 k_{1}} d_{0}\left(\varphi_{0}, M_{0}(s, x)\right) e^{-\frac{\lambda_{1} t}{2}}, t \geq 0 .
\end{aligned}
$$

Therefore

$$
u\left(t, x ; \varphi_{0}\right) \in M\left(t, x, \sqrt{2 k_{1}} d_{0}\left(\varphi_{0}, M_{0}(s, x)\right) e^{-\frac{\lambda_{1} t}{2}}\right), \varphi_{0} \in P C B, x \in \Theta, t \geq 0,
$$

which proves the global exponential stability of the integral manifold $M$.

The results in theorems 3 and 4 extend and generalize the existing stability results for single solutions for impulsive reaction-diffusion CGNNs $[34,35,37-40]$ to the integral manifolds case. The new stability results are obtained for the set $\Theta=\prod_{q=1}^{n}\left[a_{q}, b_{q}\right]$, where $a_{q}, b_{q} \in \mathbb{R}, \mathbf{0} \in \Theta$, and can be easily applied to the most studied particular case, when the set $\Theta$ of points $x, x=\left(x_{1}, x_{2}, \ldots, x_{n}\right)^{T}$ is such that $\left|x_{q}\right|<l_{q}, l_{q}>0, q=1,2 \ldots, n$.

\section{Examples and Discussions}

Example 1. Consider a delayed impulsive reaction-diffusion CGNN model with variable impulsive perturbations of type (1), for $n=m=2, \Theta \subset \mathbb{R}^{2}, \Theta=[0,1] \times[0,2]$ given by

$$
\left\{\begin{aligned}
\frac{\partial u_{i}(t, x)}{\partial t}= & \sum_{q=1}^{2} \frac{\partial}{\partial x_{q}}\left(D_{i q} \frac{\partial u_{i}(t, x)}{\partial x_{q}}\right)-a_{i}\left(u_{i}(t, x)\right)\left[b_{i}\left(u_{i}(t, x)\right)\right. \\
& -\sum_{j=1}^{2} c_{i j}(t) f_{j}\left(u_{j}(t, x)\right) \\
& \left.-\sum_{j=1}^{2} w_{i j}(t) g_{j}\left(u_{j}\left(t-s_{j}(t), x\right)\right)\right], t \neq \tau_{k}(u(t, x)), k=1,2, \ldots, \\
u\left(t^{+}, x\right)- & u(t, x)=\left(\begin{array}{rr}
1-\frac{1}{2 k} & 0 \\
0 & 1-\frac{1}{3 k}
\end{array}\right) u(t, x), t=\tau_{k}(u(t, x)), k=1,2, \ldots,
\end{aligned}\right.
$$


where $t>0, \tau_{k}\left(u_{i}\right)=\left|u_{i}\right|+k, k=1,2, \ldots, f_{i}\left(u_{i}\right)=g_{i}\left(u_{i}\right)=\frac{1}{2}\left(\left|u_{i}+1\right|-\left|u_{i}-1\right|\right), s_{1}(t)=s_{2}(t)=$ $e^{t} /\left(1+e^{t}\right), 0 \leq s_{i}(t) \leq v(v=1), a_{i}\left(u_{i}\right)=1, b_{1}\left(u_{i}\right)=2 u_{i}, b_{2}\left(u_{i}\right)=u_{i}, i=1,2$,

$$
\begin{gathered}
\left(c_{i j}\right)(t)=\left(\begin{array}{ll}
c_{11}(t) & c_{12}(t) \\
c_{21}(t) & c_{22}(t)
\end{array}\right)=\left(\begin{array}{ll}
0.5-0.2 \cos (t) & 0.5-0.1 \sin (t) \\
0.6-0.4 \sin (t) & 0.3-0.2 \cos (t)
\end{array}\right), \\
\left(w_{i j}\right)(t)=\left(\begin{array}{ll}
w_{11}(t) & w_{12}(t) \\
w_{21}(t) & w_{22}(t)
\end{array}\right)=\left(\begin{array}{ll}
0.1+0.3 \cos (t) & 0.2-0.3 \sin (t) \\
0.2-0.1 \sin (t) & 0.5-0.1 \cos (t)
\end{array}\right), \\
\left(D_{i q}\right)_{2 \times 2}=\left(\begin{array}{ll}
D_{11} & D_{12} \\
D_{21} & D_{22}
\end{array}\right)=\left(\begin{array}{ll}
3+\sin t & 0 \\
0 & 2+\cos t
\end{array}\right) .
\end{gathered}
$$

It is obvious that $\tau_{k}\left(u_{i}\right)$ are continuous on $\mathbb{R}$ and for $i=1,2$

$$
\tau_{1}\left(u_{i}\right)<\tau_{2}\left(u_{i}\right)<\ldots, \tau_{k}\left(u_{i}\right) \rightarrow \infty \text { as } k \rightarrow \infty .
$$

We can also verify that the assumptions A1-A7 are satisfied for $\underline{a}_{i}=\bar{a}_{i}=1, i=1,2, B_{1}=2, B_{2}=1$, $L_{1}=L_{2}=M_{1}=M_{2}=1$ and

$$
\left(d_{i q}\right)_{2 \times 2}=\left(\begin{array}{ll}
d_{11} & d_{12} \\
d_{21} & d_{22}
\end{array}\right)=\left(\begin{array}{ll}
2 & 0 \\
0 & 1
\end{array}\right) .
$$

In addition, we have that $B=2$ and $\underline{D}=1$.

Consider the manifold

$$
M=[-1, \infty) \times \Theta \times\left\{u \in \mathbb{R}_{+}^{2}: u \leq u^{C}\right\},
$$

where $u^{C}=\left(u_{1}^{C}, u_{2}^{C}\right)^{T}$ is a constant solution of the model (26). The existence of an equilibrium $u^{C}$ of the impulsive delayed reaction-diffusion CGNN model (26) is guaranteed by conditions A1-A7 and the assumptions on the impulsive functions and hypersurfaces.

Finally, we have that condition (4) of Theorem 1 holds for

$$
c_{1}=\min _{1 \leq i \leq 2}\left[2\left(\frac{4 n \underline{D}}{B^{2}}+\underline{a}_{i} B_{i}\right)-\bar{a}_{i} \sum_{j=1}^{2}\left(L_{j} \bar{c}_{i j}+M_{j} \bar{w}_{i j}+L_{i} \bar{c}_{j i}\right)\right]=2.4
$$

and

$$
c_{2}=\max _{1 \leq i \leq 2}\left(M_{i} \sum_{j=1}^{2} \bar{a}_{j} \bar{w}_{j i}\right)=1.1
$$

and

$$
\gamma_{1 k}=1-\frac{1}{2 k}, \gamma_{2 k}=1-\frac{1}{3 k}, k=1,2, \ldots
$$

Therefore, according to Theorem 1, the manifold $M$ defined by (27) is an integral manifold of (26), and by Theorem 2, we conclude that it is uniformly bounded. The global exponential stability of the integral manifold $M$ follows from Theorem 4 for $\lambda_{1}$ such that $0<\lambda_{1} \leq 1.3$.

In the above example, since

$$
\gamma_{1 k}=1-\frac{1}{2 k}, \gamma_{2 k}=1-\frac{1}{3 k}, k=1,2, \ldots,
$$

by means of the impulsive control the stability properties of the system without impulsive perturbations are preserved. If the impulsive functions do not satisfy condition A7, then due to the impulsive jumps 
the stable neuronal behavior can be changed momentarily. Thus, our results offer an insight on the effects of impulsive stability and control strategies on the interactions of neurons.

Example 2. In this Example, we consider the system (26) with $m=2, n=1, \Theta=[-1,1], a_{1}=a_{2}=1$, $b_{1}\left(u_{i}\right)=b_{2}\left(u_{i}\right)=5.3 u_{i}, D_{11}=D_{21}=d_{11}=d_{21}=1, f_{i}\left(u_{i}\right)=g_{i}\left(u_{i}\right)=\tanh \left(u_{i}\right), s_{i}(t)=e^{t} / 1+e^{t}$, $i=1,2$,

$$
\begin{gathered}
C=\left(c_{i j}\right)_{2 \times 2}=\left(\begin{array}{ll}
c_{11} & c_{12} \\
c_{21} & c_{22}
\end{array}\right)=\left(\begin{array}{lr}
2 & -0.1 \\
-5 & 4.5
\end{array}\right), \\
W=\left(w_{i j}\right)_{2 \times 2}=\left(\begin{array}{ll}
w_{11} & w_{12} \\
w_{21} & w_{22}
\end{array}\right)=\left(\begin{array}{rr}
-1.5 & -0.1 \\
-0.2 & -4
\end{array}\right),
\end{gathered}
$$

and $\tau_{k}\left(u_{i}\right)=\left|u_{i}\right|+k, i=1,2, k=1,2, \ldots$, as a control system for the corresponding nonimpulsive system.

Consider the manifold

$$
M=[-1, \infty) \times \Theta \times\left\{u \in \mathbb{R}_{+}^{2}: u=u^{*}\right\},
$$

where $u^{*}=\left(u_{1}^{*}, u_{2}^{*}\right)^{T}=\left(0.3 \sin ^{2}(\pi x), 0.6 \sin ^{2}(\pi x)\right)^{T}[55]$.

Since conditions of Theorem 1 are satisfied for $c_{1}=1.9, c_{2}=1.7$ and conditions of Theorem 4 are satisfied for $0<\lambda_{1} \leq 0.2$, then the integral manifold $M$ is globally exponentially stable. This means that the master system (without impulses) and the impulsive response system are globally exponentially synchronized. Therefore, the proposed stability results can be applied as an impulsive synchronization strategy to many practical problems.

Remark 1. In the last example we extend the image encryption scheme proposed by [55] considering Cohen-Grossberg type reaction-diffusion delayed neural network and variable impulsive perturbations. The functions $b_{i}$ play the role of feedback gains in the synchronization mechanism. Thus we again demonstrate the great opportunities for applications of our results.

\section{Conclusions}

In this paper, the integral manifolds technique is applied to propose boundedness and stability criteria for a class of impulsive delayed reaction-diffusion CGNNs. The proposed results complement and extend some existing qualitative results for such models [34,35,37-40]. The consideration of variable impulsive perturbations, as well as, the use of a Poincarè-type integral inequality additionally increase the degree of generality. Two examples are provided to illustrate the proposed integral manifold method. The demonstrated integral manifold approach can be extended in the investigation of different classes of neural network and related systems. Our future studies will be focused on the consideration of systems with distributed delays and non-instantaneous impulses based on this study. Considering the case of anti-diffusion is also an important and interesting topic for future investigations.

Author Contributions: Conceptualization, G.S. and I.S.; methodology, G.S., I.S. and G.V.; formal analysis, G.S., I.S., G.V., T.S.; C.S.; investigation, G.S., I.S., G.V., C.S., T.S.; writing-original draft preparation, T.S. All authors have read and agreed to the published version of the manuscript.

Funding: This research received no external funding.

Conflicts of Interest: The authors declare no conflict of interest.

\section{References}

1. Cohen, M.A.; Grossberg, S. Absolute stability of global pattern formation and parallel memory storage by competitive neural networks. IEEE Trans. Syst. Man Cybern. 1983, 13, 815-826. [CrossRef]

2. Guo, S.; Huang, L. Stability analysis of Cohen-Grossberg neural networks. IEEE Trans. Neural Netw. 2006, 17, 106-117. [CrossRef]

3. Meng, Y.; Huang, L.; Guo, Z.; Hu, Q. Stability analysis of Cohen-Grossberg neural networks with discontinuous neuron activations. Appl. Math. Model. 2010, 34, 358-365. [CrossRef] 
4. Wan, A.; Wang, M.; Peng, J.; Mao, W. Global exponential stability analysis of Cohen-Grossberg neural networks. Math. Appl. 2006, 19, 381-387.

5. Gan, Q. Adaptive synchronization of Cohen-Grossberg neural networks with unknown parameters and mixed time-varying delays. Commun. Nonlinear Sci. Numer. Simul. 2012, 17, 3040-3049. [CrossRef]

6. Song, Q.; Cao, J. Stability analysis of Cohen-Grossberg neural network with both time-varying and continuously distributed delays. Comput. Appl. Math. 2006, 197, 188-203. [CrossRef]

7. Yuan, K.; Cao, J.; Li, H. Robust stability of switched Cohen-Grossberg neural networks with mixed time-varying delays. IEEE Trans. Syst. Man Cybern. 2006, 36, 1356-1363. [CrossRef] [PubMed]

8. Aouiti, C.; Assali, E.A. Nonlinear Lipschitz measure and adaptive control for stability and synchronization in delayed inertial Cohen-Grossberg-type neural networks. Int. J. Adapt. Control 2019. [CrossRef]

9. Ozcan, N. Stability analysis of Cohen-Grossberg neural networks of neutral-type: Multiple delays case. Neural Netw. 2019, 113, 20-27. [CrossRef] [PubMed]

10. Pratap, K.A.; Raja, R.; Cao, J.; Lim, C.P.; Bagdasar, O. Stability and pinning synchronization analysis of fractional order delayed Cohen-Grossberg neural networks with discontinuous activations. Appl. Math. Comput. 2019, 359, 241-260. [CrossRef]

11. Chen, W.H.; Liu, L.; Lu, X. Intermittent synchronization of reaction-diffusion neural networks with mixed delays via Razumikhin technique. Nonlinear Dyn. 2017, 87, 535-551. [CrossRef]

12. Lu, J.G. Global exponential stability and periodicity of reaction-diffusion delayed recurrent neural networks with Dirichlet boundary conditions. Chaos Solitons Fractals 2008, 35, 116-125. [CrossRef]

13. Qiu, J. Exponential stability of impulsive neural networks with time-varying delays and reaction-diffusion terms. Neurocomputing 2007, 70, 1102-1108. [CrossRef]

14. Rakkiyappan, R.; Dharani, S.; Zhu, Q. Synchronization of reaction-diffusion neural networks with time-varying delays via stochastic sampled-data controller. Nonlinear Dyn. 2015, 79, 485-500. [CrossRef]

15. Stamova, I.M.; Simeonov, S. Delayed reaction-diffusion cellular neural networks of fractional order: Mittag-Leffler stability and synchronization. J. Comput. Nonlinear Dynam. 2018, 13, 011015-1. [CrossRef]

16. Li, R; Cao, J.; Alsaedi, A.; Ahmad, B. Passivity analysis of delayed reaction-diffusion Cohen-Grossberg neural networks via Hardy-Poincarè inequality. J. Frankl. Inst. 2017, 354, 3021-3038. [CrossRef]

17. Wang, Z.; Zhang, H. Global asymptotic stability of reaction-diffusion Cohen-Grossberg neural networks with continuously distributed delays. IEEE Trans. Neral Netw. 2010, 21, 39-49. [CrossRef]

18. Yan, P.; Lv, T. Periodicity of delayed reaction-diffusion high-order Cohen-Grossberg neural networks with Dirichlet boundary conditions. Rocky Mountain J. Math. 2011, 41, 949-970. [CrossRef]

19. Zhao, H.; Wang, K. Dynamical behaviors of Cohen-Grossberg neural networks with delays and reaction-diffusion terms. Neurocomputing 2006, 70, 536-543. [CrossRef]

20. Benchohra, M.; Henderson, J.; Ntouyas, J. Impulsive Differential Equations and Inclusions, 1st ed.; Hindawi Publishing Corporation: New York, NY, USA, 2006; ISBN 977594550X; 978-9775945501.

21. Haddad, W.M.; Chellaboina, V.S.; Nersesov, S.G. Impulsive and Hybrid Dynamical Systems, Stability, Dissipativity, and Control, 1st. ed.; Princeton University Press: Princeton, NJ, USA, 2006; ISBN 9780691127156.

22. Li, X.; Bohner, M.; Wang, C.K. Impulsive differential equations: Periodic solutions and applications. Autom. J. IFAC 2015, 52, 173-178. [CrossRef]

23. Li, X.; Wu, J. Sufficient stability conditions of nonlinear differential systems under impulsive control with state-dependent delay. IEEE Trans. Automat. Control 2018, 63, 306-311. [CrossRef]

24. Liu, X.; Zhang, K. Impulsive Systems on Hybrid Time Domains, 1st ed.; Springer: Cham, Switzerland, 2019; ISBN 978-3-030-06211-8; 978-3-030-06212-5.

25. Stamova, I.M. Impulsive control for stability of n-species Lotka-Volterra cooperation models with finite delays. Appl. Math. Lett. 2010, 23, 1003-1007. [CrossRef]

26. Stamova, I.M.; Stamov, A.G. Impulsive control on the asymptotic stability of the solutions of a Solow model with endogenous labor growth. J. Frankl. Inst. 2012, 349, 2704-2716. [CrossRef]

27. Stamova, I.M.; Stamov, G.T. Applied Impulsive Mathematical Models, 1st ed.; Springer: Cham, Switzerland, 2016; ISBN 978-3-319-28060-8; 978-3-319-28061-5.

28. Stamova, I.; Stamov, G. Mittag-Leffler synchronization of fractional neural networks with time-varying delays and reaction-diffusion terms using impulsive and linear controllers. Neural Netw. 2017, 96, 22-32. [CrossRef] [PubMed] 
29. Yang, X.; Peng, D.; Lv, X.; Li, X. Recent progress in impulsive control systems. Math. Comput. Simul. 2019, 155, 244-268. [CrossRef]

30. Aouiti, C.; Dridi, F. New results on impulsive Cohen-Grossberg neural networks. Neural Proc. Lett. 2019, 49, 1459-1483. [CrossRef]

31. Bohner, M.; Stamov, G.T.; Stamova, I.M. Almost periodic solutions of Cohen-Grossberg neural networks with time-varying delay and variable impulsive perturbations. Commun. Nonlinear Sci. Numer. Simul. 2020, 80, 104952. [CrossRef]

32. Li, X. Exponential stability of Cohen-Grossberg-type BAM neural networks with time-varying delays via impulsive control. Neurocomputing 2009, 73, 525-530. [CrossRef]

33. Li, X. Existence and global exponential stability of periodic solution for impulsive Cohen-Grossberg-type BAM neural networks with continuously distributed delays. Appl. Math. Comput. 2009, 215, 292-307. [CrossRef]

34. Li, Z.; Li, K. Stability analysis of impulsive Cohen-Grossberg neural networks with distributed delays and reaction-diffusion terms. Appl. Math. Model. 2009, 33, 1337-1348. [CrossRef]

35. Li, K.; Song, Q. Exponential stability of impulsive Cohen-Grossberg neural networks with time-varying delay and reaction-diffusion terms. Neurocomputing 2008, 72, 231-240. [CrossRef]

36. Li, Y.; Zhang, T. Global exponential stability and existence of anti-periodic solutions to impulsive Cohen-Grossberg neural networks on time scales. Topol. Methods Nonlinear Anal. 2015, 45, 363-384.

37. Pan, J.; Liu, X.; Zhong, S. Stability criteria for impulsive reaction-diffusion Cohen-Grossberg neural networks with time-varying delays. Math. Comput. Model. 2010, 51, 1037-1050. [CrossRef]

38. Wu, C. Existence of periodic solutions for Cohen-Grossberg neural networks with time-varying delays and impulses. In Advances in Neural Networks, 1st ed.; Liu, D., Zhang, H., Polycarpou, M., Alippi, C., He, H., Eds.; Springer: Berlin/Heidelberg, Germany, 2011; Volume 6675, pp. 521-528, ISBN 978-3-642-21104-1.

39. Wang, J.L.; Wu, H.N.; Guo, L. Stability analysis of reaction-diffusion Cohen-Grossberg neural networks under impulsive control. Neurocomputing 2013, 106, 21-30. [CrossRef]

40. Zhang, X.; Wu, S.; Li, K. Delay-dependent exponential stability for impulsive Cohen-Grossberg neural networks with time-varying delays and reaction-diffusion terms. Commun. Nonlinear Sci. Numer. Simul. 2011, 16, 1524-1532. [CrossRef]

41. Akhmet, M. Integral manifolds of differential equations with piecewise constant argument of generalized type. arXiv 2005, arXiv:math/0508230.

42. Akhmetov, M.U.; Perestyuk, N.A. Integral sets of quasilinear impulse systems. Ukrainian Math. J. 1992, 44, 1-17. [CrossRef]

43. Bogoliubov, N.N.; Mitropolsky, Y.A. The method of integral manifolds in nonlinear mechanics. Contrib. Differ. Equ. 1963, 2, 123-196.

44. Constantin, P.; Foias, C.; Nicolaenko, B.; Temam, R. Integral Manifolds and Inertial Manifolds for Dissipative Partial Differential Equations, 1st ed.; Springer: New York, NY, USA, 1989; ISBN 0-387-96729-X.

45. Hale, J.K.; Stokes, A. Behaviour of solutions near integral manifolds. Arch. Rational Mech. Anal. 1960, 6, 133-170. [CrossRef]

46. Mitropol'skiy, Y.A. The method of integral manifolds in the theory of nonlinear oscillations. In International Symposium on Nonlinear Differential Equations and Nonlinear Mechanics, 1st ed.; Lasalle, J., Ed.; Academic Press: New York, NY, USA, 1963; pp. 1-15, ISBN 9780323147309.

47. Stamov, G.T.; Stamova, I.M. Integral manifolds for uncertain impulsive differential-difference equations with variable impulsive perturbations. Chaos Solitons Fractals 2014, 65, 90-96. [CrossRef]

48. Stamov, G.; Stamova, I. Impulsive delayed Lasota-Wazewska fractional models: Global stability of integral manifolds. Mathematics 2019, 7, 1025. [CrossRef]

49. Benchohra, M.; Henderson, J.; Ntouyas, S.K.; Ouahab, A. Impulsive functional differential equations with variable times. Comput. Math. Appl. 2004, 47, 1659-1665. [CrossRef]

50. Song, Q.; Yang, X.; Li, C.; Huang, T.; Chen, X. Stability analysis of nonlinear fractional-order systems with variable-time impulses. J. Franklin Inst. 2017, 354, 2959-2978. [CrossRef]

51. Yang, X.; Li, C.; Song, Q.; Huang, T.; Chen, X. Mittag-Leffler stability analysis on variable-time impulsive fractional-order neural networks. Neurocomputing 2016, 207, 276-286. [CrossRef]

52. Yilmaz, E. Almost periodic solutions of impulsive neural networks at non-prescribed moments of time. Neurocomputing 2014, 141, 148-152. [CrossRef] 
53. Cheung, W.-S. Some new Poincarè-type inequalities. Bull. Austral. Math. Soc. 2001, 63, 321-327. [CrossRef]

54. Lai, X.; Yao, T. Exponential stability of impulsive delayed reaction-diffusion cellular neural networks via Poincarè integral inequality. Abstr. Appl. Anal. 2013, 2013, 131836. [CrossRef]

55. Chen, W.; Luo, S.; Zheng, W.X. Impulsive synchronization of reaction-diffusion neural networks with mixed delays and its application to image encryption. IEEE Trans. Neural Netw. Learn. Syst. 2016, 27, 2696-2710. [CrossRef]

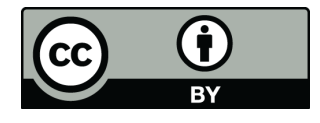

(C) 2020 by the authors. Licensee MDPI, Basel, Switzerland. This article is an open access article distributed under the terms and conditions of the Creative Commons Attribution (CC BY) license (http://creativecommons.org/licenses/by/4.0/). 